0038-1098(95)00444-0

\title{
ELASTIC WAVE BAND GAPS IN 3-D PERIODIC POLYMER MATRIX COMPOSITES
}

\author{
M. Kafesaki*, M. M. Sigalas** and E. N. Economou* \\ ${ }^{*}$ Research Center of Crete, FORTH, P.O. Box 1527, 71110 Heraklio, and \\ Department of Physics, University of Crete \\ "*Ames Laboratory and Department of Physics and Astronomy Iowa State \\ University, Ames IA 50011
}

(Received 25 May 1995; accepted 26 June 1995 by P.H. Dederichs)

\begin{abstract}
We present band structure results for elastic waves in periodic composite materials consisting of scatterers (spheres, cubes, or rods) embedded in a homogeneous polymer matrix. The material of the scatterers is a high density material (such as $\mathrm{Steel}, \mathrm{Ni}, \mathrm{Pb}, \mathrm{Cu}, \mathrm{etc}$.). In all cases, we find wide full band gaps in fcc, bcc and $\mathrm{sc}$ structures for a wide range of filling ratios. We show that the existence of these wide gaps can been analyzed and predicted by using the single scattering results.
\end{abstract}

Keywords: D. acoustic properties D. elasticity

There is a growing interest in recent years for the propagation of elastic (EL) and acoustic (AC) waves in random and periodic composite materials [1] - [14]. The interest among solid state physicists is mainly connected to the question of the existence or not of spectral gaps in periodic systems or localized waves in disordered systems in analogy with what happens to the electrons in solids. The attention to the acoustic and elastic waves in connection with gaps or localization is not only due to their many applications (in geophysics, medicine, oil exploration, etc.) $[15,16]$ but to their rich physics as well: EL waves are full vector waves with different velocity of propagation between the longitudinal and the transverse component; in the EL and $A C$ wave equation there is a term proportional to the mass density variation which may cause the appearance of novel behavior. Furthermore, ACW and ELW in structures exhibiting localized eigenwaves (i.e. mobility gaps) offer themselves for an accurate experimental investigation of open questions regarding the problem of disorder induced localization.

The difficulty to construct composites sustaining localized eigenstates, was greatly reduced following the suggestion by John and Rangarajan [17] and by Economou and Zdetsis [18]; they pointed out that the existence of bands of localized eigenstates in random systems is directly related to the existence of gaps in periodic systems since both are due to destructive interference of the multiple scattered waves. For this reason attention has been focused on the easier problem of $\mathrm{ACW}$ and ELW propagation in periodic systems for which one can employ computational methods that have been already developed for the electronic propagation in periodic crystals.
The study of acoustic and elastic wave propagation in periodic binary composites consisting of spheres embedded in a host (3-D) or from cylinders embedded in a host (2-D), shows $[4,8,9,11,12]$ that gaps can exist under rather extreme conditions. These conditions concern mainly the density and velocity contrast of the components of the composite, the volume fraction of one of the two components, the lattice structure and the topology $[11,12]$; they are realized in a $\mathrm{Be}$ or $\mathrm{Si}$ or $\mathrm{SiO}_{2}$ matrix with embedded $\mathrm{Au}$ or $\mathrm{Pb}$ spheres placed periodically in an fcc lattice [12]. For 2-D square lattice, full band gaps have been found only in Au cylinders in Be host [8]. However, the 2-D hexagonal lattices have proven more favorable for the creation of the gaps; in particular, rods from $\mathrm{Mo}, \mathrm{Al}_{2} \mathrm{O}_{3}, \mathrm{Fe}$, and steel embedded in Lucite host exhibit relatively wide gaps [11]. From the experimental point of view, Kinra and Ker [3] have measured the phase velocity of longitudinal waves through 3-D periodic polymer matrix composites as a function of frequency. Vasseur et. al. [23] studied numerically the elastic band structure of 2-D commercially available composite materials such as epoxy reinforced $\mathrm{C}$ or glass fibres and they found several extremely large complete band gaps in those systems.

Recently, considerable progress was achieved in understanding the above results and in predicting favorable material combinations [19]. Whenever the scattering crosssection from a single spherical inclusion exhibits'strong and well-separated resonances with a considerable background in between attributed to either a rigid or soft sphere (in either case the field inside the inclusion is zero), a wide gap is expected. One can understand this basic result by considering two limiting channels of propagation in a composite: one is 
using the host material and avoids the inclusions; the other is employing the inclusions and hops coherently among them by a linear combination of the resonances in analogy with the LCAO in molecules and solids. In the spectral region of high background between two well-separated resonances neither channel is operational and consequently no propagation is expected to take place. This explanation was confirmed by comparing single sphere cross-sections with band structure results under various conditions [19].

In Fig.1 we show a case ( $\mathrm{Pb}$ spherical inclusion in an epoxy host) which according to the previous reasoning is expected to exhibit as a periodic composite wide spectral gaps. Fig.1a shows the total cross-section of a transverse plane wave scattered by a $\mathrm{Pb}$ spherical inclusion embedded in epoxy. In Fig.1b the $\mathrm{Pb}$ sphere has been replaced by a rigid sphere $\left(\rho \rightarrow \infty, \mu \rightarrow \infty, \lambda \rightarrow \infty, c_{\ell} \rightarrow 0, c_{t} \rightarrow 0\right)$ where $\rho$ is the density, $\mu$ and $\lambda$ are the Lamé elastic coefficients and $c_{\ell}, c_{t}$ the longitudinal and the transverse velocities $: c_{\ell}^{2}=(\lambda+2 \mu) / \rho, c_{t}^{2}=\mu / \rho ;$ Fig.1c shows the cross-section calculated by subtracting from the $\mathrm{Pb}$ scattering amplitude the rigid sphere scattering amplitude $[26,27,19]$.

There are two broad resonances (the first is a double one) in the $\mathrm{Pb}$ sphere - epoxy matrix cross-section (Fig.1a), well separated by a region of non negligible scattering. This strong background scattering cross-section in the region between the resonances is due to the contribution of the rigid sphere as the results of the subtraction of this contribution show (Fig.1c). In the longitudinal incident wave scattering concerning the same system, the cross-sections - not pre- sented here - have almost the same form with those of Fig.1. An exception is that the first resonance is not a double one and that the peaks are a little lower. The previous reasoning suggests that a wide gap is expected between first and second resonance with its width to be narrower than the spectral distance between them. Also, the resonances are expected to coincide in position with the flat bands of the system (due to level repulsion and hybridization some deviations are expected). Indeed, as will be discussed below, this turned out to be the case. The half arrows in Fig.1a (with the $\mathrm{G}$ in between) show the position and the width of the gap and the regular arrows indicate the positions of flat bands (see Fig.2).

The single sphere scattering cross-section for the opposite case of $\mathrm{Pb}$ as matrix and epoxy as sphere was also calculated. What was found is that in the longitudinal incident wave scattering case the result of the subtraction of the corresponding background (soft sphere) is two very low resonances, while in the transverse incident wave case the cross-section is very smooth and low. These results are not favorable for gap creation. Indeed, explicit band structure calculations show no gaps in this case.

The conclusion of the above discussion is that periodic composites consisting of polymers (such as epoxy) as a host with high density metal inclusions (such as $\mathrm{Pb}$ ) are expected to produce wide spectral gaps in ELW propagation. An additional advantage is that polymer materials are easily fabricated. Below we present EL wave band structure computational results for 3-D periodic composites consisting of
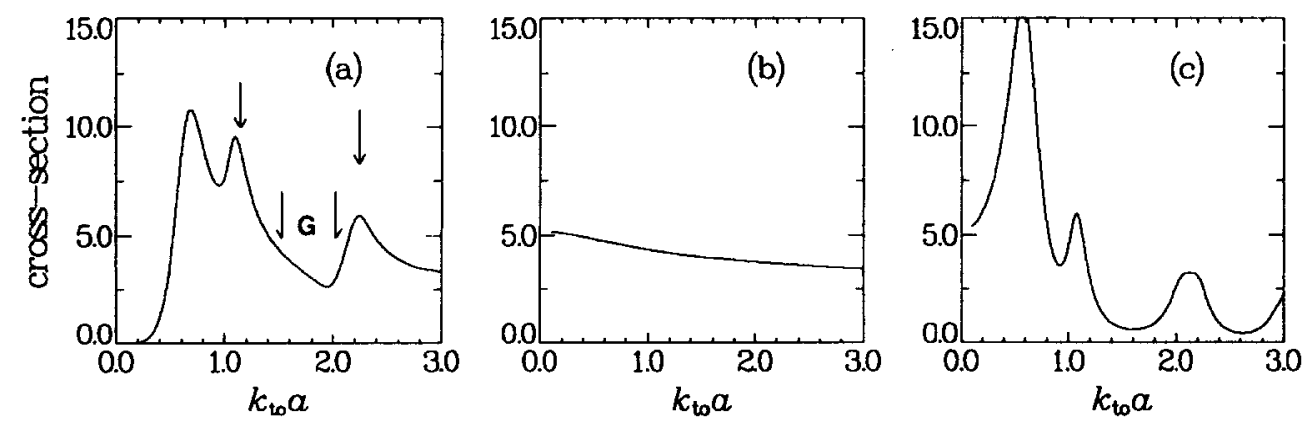

Fig.1 Total dimensionless cross-sections vs $k_{t o} a$ for $\mathrm{Pb}$ sphere in epoxy matrix (a), rigid sphere in epoxy matrix (b). The third panel (c) represents the crosssection calculated by subtracting from the $\mathrm{Pb}$ sphere scattering amplitude the rigid sphere scattering amplitude. The incident wave is transverse, $a$ is the radius of the sphere and $k_{t_{o}}=\omega / c_{t_{0}}$ the transverse wave number in the medium of the matrix. The arrows indicate the positions of the flat bands and the half arrows (with the symbol $G$ in between) the position of the gap in the corresponding fcc periodic composite with volume fraction of the spheres $x=0.262$. 


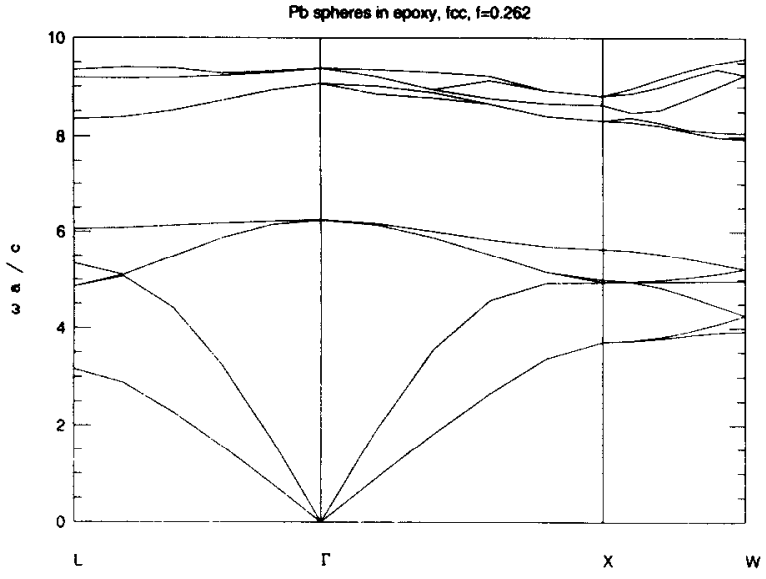

Fig.2 Elastic wave band structure for a fcc periodic composite consisting of $\mathrm{Pb}$ spheres in epoxy matrix. The volume fraction of the spheres is $0.262 ; \omega$ is the frequency, $a$ is the lattice constant and $c$ the transverse wave velocity in the epoxy $\left(c_{t o}\right)$.

inclusions formed by a high density metal such as steel, $\mathrm{Pb}$ and $W$ and embedded in epoxy.

For a locally isotropic medium, the elastic wave equation is [25]:

$$
\frac{\partial^{2} u^{i}}{\partial t^{2}}=\frac{1}{\rho}\left\{\frac{\partial}{\partial x_{i}}\left(\lambda \frac{\partial u^{\ell}}{\partial x_{\ell}}\right)+\frac{\partial}{\partial x_{\ell}}\left[\mu\left(\frac{\partial u^{i}}{\partial x_{\ell}}+\frac{\partial u^{\ell}}{\partial x_{i}}\right)\right]\right\}
$$

where $u^{i}$ is the $\mathrm{i}$-th component of the displacement vector $\vec{u}(\vec{r}) ; \lambda(\vec{r})$ and $\mu(\vec{r})$ are the Lamé coefficients [25] and $\rho(\vec{r})$ is the density. For periodic media $f(\vec{r})(f \equiv \mu, \lambda$ or $\rho^{-1}$ ) can be expanded in terms of its Fourier components $f_{G}$. The displacement vector $\vec{u}(\vec{r})$ which satisfies Bloch's theorem can be expanded in terms of plane waves: $\vec{u}(\vec{r})=\sum_{\vec{K}} \vec{u}_{\vec{K}} e^{i \vec{K} \vec{r}}$ where $\vec{K}=\vec{k}+\vec{G}$, and the summation is over $\vec{G}$. Keeping $\mathrm{N}$ reciprocal vectors, $\vec{G}$, in the previous sum and substituting in Eq.(2.1), we get a $3 N \times 3 N$ matrix eigenvalue equation for the $3 \mathrm{~N}$ unknown coefficients $\vec{u}_{\vec{K}}$. The number $\mathbf{N}$ is increased until the desired convergence is achieved. In the present calculations we kept $\mathrm{N}=400 \vec{G}$ vectors to achieve convergence better than $5 \%$.

Fig.2 shows the band structure of elastic waves propagating in an fcc lattice consisting of $\mathrm{Pb}$ spheres $(\rho=$ $\left.11.357 \mathrm{~g} / \mathrm{cm}^{3}, c_{\ell}=2.158 \mathrm{~km} / \mathrm{s} c_{t}=0.860 \mathrm{~km} / \mathrm{s}\right) \mathrm{em}$ bedded in epoxy $\left(\rho=1.180 \mathrm{~g} / \mathrm{cm}^{3}, c_{\ell}=2.540 \mathrm{~km} / \mathrm{s}\right.$ $c_{t}=1.160 \mathrm{~km} / \mathrm{s}$ ); the radius of the spheres is 0.25 of the lattice constant corresponding to filling ratio 0.262 . There is a complete band gap for all the directions in the Brillouin zone between the 6 th and 7 th bands. The lower edge of the gap is at $\Gamma$ point, the upper edge of the gap is at $W$ point and the width of the gap over the midgap frequency, $\Delta \omega / \omega_{g}$, is 0.24 .

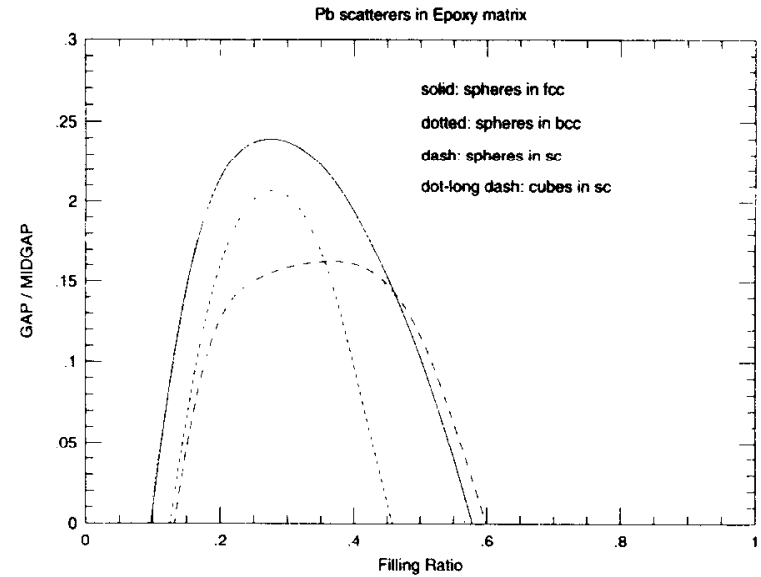

Fig. 3 The width of the gap over the midgap frequency vs filling ratio for a periodic composite consisting of $\mathrm{Pb}$ spheres in epoxy matrix and fcc (solid line), bcc (dotted line) and sc (dashed line) structure. The dotted-long dashed line corresponds to $\mathrm{Pb}$ cubes in epoxy matrix and sc structure.

Fig.3 shows the $\Delta \omega / \omega_{g}$ vs the filling ratio for a $\mathrm{Pb}$ spheres-epoxy matrix system for three different structures: fcc, bcc, and sc. The curves are almost the same for both fcc and bcc structures; the maximum $\Delta \omega / \omega_{g}$ is almost 0.24 at filling ratios around 0.25 . For sc structure, the maximum $\Delta \omega / \omega_{g}$ is 0.2 at filling ratio 0.28 . The gap appears for filling ratios between 0.1 and 0.57 for both fcc and bcc structures while for sc structure there is gap for filling ratios between 0.13 and 0.46 . This is consistent with the empirical observation which states that the cermet topology is better for gap appearance than the network topology $[10,11]$. In the case of spherical scatterers, the cermet topology exists for filling ratios less than $0.74,0.68$, and 0.52 in fcc, bcc, and sc structures respectively (for filling ratios greater than those values the spheres overlap which corresponds to network topology). Thus, in sc structures the transition from cermet to network topology appears at smaller filling ratios than for both fcc and bec structures.

Comparing the results of the sc structure with scatterers either cubes or spheres, we found that the maximum value of $\Delta \omega / \omega_{g}$ appears for spheres. On the other hand, the gaps appear in a wider range of filling ratios for the case of cubes. This can be explained by noting that the transition from the cermet to network topology appears at the filling ratio 1 (the cubes are touching each other only in the extreme case where the edge is equal to the lattice constant).

There are no gaps in systems consisting of isolated epoxy scatterers in $\mathrm{Pb}$ background which is in accordance with the previously mentioned requirements for the appearance of gaps. We also studied structures consisting of tetragonal rods connecting nearest neighbors in sc lattice [24]. We did not find any gaps for either $\mathrm{Pb}$ rods in epoxy or epoxy 
rods in $\mathrm{Pb}$ background. This is further support the empirical requirement which states that the cermet topology is more favorable for the appearance of the gaps $[10,11]$.

Full band gaps were found for several other systems. In all the cases, the fcc structures give slightly wider band gaps than the bcc structures while the sc lattices give even smaller gaps. Plastics, such as epoxy or PMMA, are ideal materials for the background medium and the maximum value of $\Delta \omega / \omega_{g}$ appeared for filling ratios of the isolated spherical scatterers between 0.25 to 0.5 . As scatterer material, we used $\mathrm{W}, \mathrm{Ni}, \mathrm{Fe}, \mathrm{Cu}$, Steel, and $\mathrm{Ag}$. The densities and velocities of these materials as well as the maximum value of $\Delta \omega / \omega_{g}$ for epoxy host and fcc lattice are given in the following table.

$\begin{array}{lccrc} & c_{\ell}(\mathrm{km} / \mathrm{s}) & c_{t}(\mathrm{~km} / \mathrm{s}) & \rho\left(\mathrm{g} / \mathrm{cm}^{3}\right) & \max \left(\Delta \omega / \omega_{g}\right) \\ \mathrm{W} & 5.233 & 2.860 & 18.700 & 0.75 \\ \mathrm{Ni} & 5.894 & 3.219 & 8.968 & 0.55 \\ \mathrm{Fe} & 6.064 & 3.325 & 7.860 & 0.50 \\ \mathrm{Cu} & 4.726 & 2.298 & 8.960 & 0.50 \\ \mathrm{Steel} & 5.940 & 3.220 & 7.800 & 0.50 \\ \mathrm{Ag} & 3.789 & 1.950 & 10.635 & 0.50\end{array}$

For all these systems the gap appears between the 6th and 7 th bands. Also, the scatterer is the high density material in accordance with the previous experience. On the other hand, the velocities of the scatterers are either smaller (for the $\mathrm{Pb}$ ) or higher (for all the other materials) than those of the matrix material. From the present results, it seems that high density scatterers in a low density background is the most important condition for the appearance of gaps.

It is worth mentioning that experiments on two systems similar with those that we have studied in the present work have already been done [3]. The first system consists of Steel spheres embedded in plastic (PMMA) forming a tetragonal lattice and the second one consists of Glass spheres in plastic (epoxy) forming a sc lattice. The results from our calculations for the dispersion curve of the longitudinal-like waves (these are the only modes that have been measured in the experiments) are in good agreement with the measurements (the differences are less than $15 \%$ ) However, our calculations show that none of those systems exhibit a full band gap, although, in particular for the Steel in PMMA system, full band gaps can be found in sc structures instead the tetragonal structure that they used in the original experiment [3].

In all examined cases (epoxy as host and $\mathrm{W}, \mathrm{Ag}, \mathrm{Fe}, \mathrm{Ni}$, $\mathrm{Cu}$ as sphere material) for which wide gaps where found, the single scattering study gave cross-sections similar to that shown in Fig.1 with the two broad resonances and the noticeable contribution of the background between them. In all these cases the single-scattering study gave also good estimations for the position of the gap as obtained from the corresponding band structure calculations.

Finally, the above mentioned as well as additional single scattering results, strongly indicate that the density contrast of the two materials (scatterer - matrix) is a much more important parameter for the appearance of a gap than the velocity contrast.

Acknowledgment - Ames Laboratory is operated by the U.S. Department of Energy by Iowa State University under Contract No. W-7405-Eng-82.

\section{REFERENCES}

[1] P. Sheng, ed., Scattering and Localization of Classical Waves in Random Media, (World Scientific, Singapore, 1990).

[2] See the proceedings of the NATO ARW, Photonic Band Gaps and Localization, ed. C. M. Soukoulis, (Plenum, New York, 1993).

[3] V. K. Kinra and E. L. Ker, Int. J. Solids Structures 19, 393 (1983).

[4] M.M. Sigalas and E.N. Economou, J. Sound Vibration 158, 377 (1992).

[5] J. P. Dowling, J. Acoust. Soc. Am. 91, 2539 (1992).

[6] L. Ye, G. Cody, M. Zhou, P. Sheng, and A. N. Norris, Phys. Rev. Lett. 69, 3080 (1992).

[7] M.S. Kushwaha, P. Halevi, L. Xobrzynski, and B. Djafari-Rouhani, Phys. Rev. Lett. 71, 2022 (1993).

[8] M.M. Sigalas and E.N. Economou, Solid State Commun. 86, 141 (1993).
[9] E.N. Economou and M.M. Sigalas, in Photonic Band Gaps and Localization ed. by C.M. Soukoulis (Plenum Press, New York, 1993), pp. 317-338.

[10] E.N. Economou and M.M. Sigalas, Phys. Rev. B 48, 13434 (1993).

[11] M.M. Sigalas, E.N. Economou, and M. Kafesaki, Phys. Rev. B 50, 3393 (1994).

[12] E.N. Economou and M.M. Sigalas, J. Acoust. Soc. Am. 95, 1734 (1994).

[13] M.S. Kushwaha, and P. Halevi, Appl. Phys. Lett. 64, 1085 (1994).

[14] M.S. Kushwaha, P. Halevi, G. Martinez, L. Xobrzynski, and B. Djafari-Rouhani, Phys. Rev. B 49, 2313 (1994).

[15] W. A. Smith and B. A. Auld, IEEE Trans. Ultrason. Ferroelectrics, Frequency Control 38, 40 (1991).

[16] B. A. Auld, Y. A. Shui, and Y. Wang, J. de Physique, Colloque C5, suppl. No.4, 45 (1984). 
[17] S. John and R. Rangarajan, Phys. Rev. B 38, 10101 (1988).

[18] E. N. Economou and A. Zdetsis, Phys. Rev. B 40, 1334 (1989).

[19] M. Kafesaki and E. N. Economou, unpublished.

[20] S. John, Physics Today 40, 32 (1991); Philip St. J. Russel, Physics World 37, August 1992; J. Maddox, Nature 348, 481 (1990).

[21] See the special issue of the J. Opt. Soc. Amer. B 10, 208-408 (1993) on Development and Applications of Materials Exhibiting Photonic Band Gaps.

[22] S. John, Phys. Rev. Lett. 58, 2486 (1987).
[23] J. O. Vasseur, B. Djafari-Rohani, L. Xobrzynski, M.S. Kushwaha, and P. Halevi, J. Phys..Condens. Matter 6, 8759 (1994).

[24] H. S. Sozuer and J. W. Haus, J. Opt. Soc. Am. B 10, 296 (1993).

[25] Landau, L. D., and Lifshitz, E. M., 1959, “Theory of Elasticity", (Pergamon, London, 1959).

[26] G. Gaunaurd and H. Uberall, J. Acoust. Soc. Am. 67, 1432 (1980).

[27] L. Flax, G. Gaunaurd and H. Uberall, in Physical Acoustics, edited by W. P. Mason (Academic, New York, 1981), vol XV. 